\title{
THE ATTRIBUTES DIAGRAM \\ A Geometrical Framework for Assessing the Quality of Probability Forecasts *
}

\author{
Wu-ron HSU
}

National Taiwan University, Taipei, Taiwan, ROC

\author{
Allan H. MURPHY \\ Oregon State University, Corvallis, OR 97331, USA
}

\begin{abstract}
In two-event situations, a reliability diagram provides a geometrical framework for evaluating this attribute of probability forecasts. However, reliability is only one of several important attributes of such forecasts. This paper describes an extension of the reliability diagram - the attributes diagram - in which the accuracy, resolution, and skill, as well as the reliability, of probability forecasts can be depicted. Moreover, these geometrical representations are shown to be directly related to quantitative measures of the respective attributes. The interpretation and use of the attributes diagram is illustrated by considering samples of probabilistic quantitative precipitation forecasts. Some possible extensions of this diagram to multiple-event situations are briefly discussed.
\end{abstract}

Keywords: Evaluation of probability forecasts, Attributes of probability forecasts, Geometrical interpretations of attributes of probability forecasts, Attributes diagram, Evaluation measures for probability forecasts, Quadratic score, Probability forecasts.

\section{Introduction}

In probability forecasting, it is important to determine the degree to which the forecast probabilities possess certain desirable properties such as reliability. Probability forecasts are reliable (or calibrated) if the relative frequency of occurrence of events assigned a forecast probability $p$ tends to be close to $p$. The reliability of objective and subjective probability forecasts has been investigated in several different contexts [e.g., Fischhoff and MacGregor (1982), Lichtenstein et al. (1982), Murphy (1985), Murphy and Brown (1984), Murphy and Winkler (1977, 1984), Wallsten and Budescu (1983)]. Moreover, reliability diagrams - in which observed relative frequency is plotted against forecast probability for specific probability values (or ranges of values) and compared with a perfect reliability line - have been used to display and evaluate the reliability of probability forecasts. These diagrams appear to provide a useful adjunct to other methods (e.g., scoring rules) of assessing the quality of probability forecasts.

Reliability is only one of several desirable properties or attributes of probability forecasts. Constant forecasts of the long-term historical probabilities would generally be quite reliable, but they would be deficient in many important respects. Other relevant attributes of probability forecasts

\footnotetext{
* This research was supported in part by the National Science Foundation (Division of Atmospheric Sciences) under grants ATM-8004680, ATM-8209713, and ATM-8507495.
} 
include accuracy, resolution, and skill (see section 2), and quantitative measures of these attributes as well as of reliability - have been formulated and used by several individuals [e.g., Murphy (1972a, b, 1973), Sanders (1963), Yates (1982)].

The primary purpose of this paper is to describe an 'attributes diagram' in which it is possible to assess the degree to which samples of probability forecasts possess certain desirable properties. We believe that this diagram will prove to be even more useful than the reliability diagram in the evaluation of probability forecasts in two-event situations. ${ }^{1}$ The attributes of probability forecasts of interest in this paper and some measures of these attributes are defined in section 2 . These measures provide quantitative descriptions of the attributes and also contribute to the interpretation of the attributes diagram. The construction of the attributes diagram itself is described in section 3 , which also illustrates the interpretation of the diagram by considering specific samples of probabilistic weather forecasts. Section 4 briefly discusses the practical use and further extension of the attributes diagram.

\section{Some attributes of probability forecasts: Definitions and quantitative measures}

Consider a sample of $K$ probability forecasts for a two-event situation (e.g., the occurrence or non-occurrence of some event). In such a situation, it is sufficient to consider only the probability assigned to the occurrence of the event. Let $r_{k}$ denote this probability on the $k$ th occasion $\left(0 \leq r_{k} \leq 1, k=1, \ldots, K\right)$. The average quadratic or Brier score $Q R$ [see Winkler and Murphy (1968), Murphy and Daan (1985)] is an overall measure of the quality of such forecasts, and $Q R$ can be defined as follows:

$Q R=(1 / K) \sum_{k=1}^{K}\left(r_{k}-d_{k}\right)^{2}$,

where $d_{k}=1$ if the event occurs on the $k$ th occasion and $d_{k}=0$ otherwise. The measure $Q R$ possesses several desirable properties, including the fact that it is a strictly proper scoring rule. Moreover, since $Q R$ provides a quantitative assessment of the average degree of correspondence between individual forecasts and observations (e.g., observed events), it is a measure of the accuracy of the forecasts [see Murphy and Daan (1985)].

Suppose that the probability $r_{k}$ can assume only a finite set of $T$ distinct values, $r_{t}$ say, or equivalently, that the range of probability values is divided into $T$ mutually exclusive and collectively exhaustive intervals, each of which is represented by a 'typical' probability value $r_{t}(t=1, \ldots, T)$. Then the sample of $K$ forecasts can be divided into $T$ subsamples, where subsample $t$ consists of the $K_{t}$ forecasts for which $r_{k}=r_{t}\left(0 \leq r_{t} \leq 1 ; \sum_{t} \mathrm{~K}_{t}=K ; t=1, \ldots, T\right)$. In this context, $Q R$ can be partitioned or decomposed into three terms as follows [Murphy (1973)]:

$Q R=\bar{d}(1-\bar{d})+(1 / K) \sum_{t=1}^{T} K_{t}\left(r_{t}-\bar{d}_{t}\right)^{2}-(1 / K) \sum_{t=1}^{r} K_{t}\left(\bar{d}_{t}-\bar{d}\right)^{2}$

in which $\bar{d}_{t}$ is the relative frequency of occurrence of the event in question when $r_{k}=r_{t}$ and $\bar{d}$ is the overall relative frequency of occurrence of the event [i.e., $\bar{d}=(1 / K) \sum_{t} K_{t} \bar{d}_{t}$ ].

The first term on the right-hand side (RHS) of (2) can be interpreted as follows: (a) the variance of the observations $\left(d_{k}\right)$ on the $K$ occasions; or (b) the quadratic score for a constant forecast of the

1 An earlier version of the attributes diagram was described by Hsu (1982). 
sample relative frequency $(\bar{d})$ over the $K$ occasions. We adopt the latter interpretation in this paper and denote the first term by $Q R^{*}$. It should also be noted here that this term does not depend on the forecasts in any way.

The second term on the RHS of (2) is the average squared difference between the forecast probability $r_{t}$ and the corresponding relative frequency $\bar{d}_{t}$. Thus, this term is a measure of the reliability (or calibration) of the forecasts, and we shall denote it by $R E L$. Note that $R E L=0$ for perfectly reliable forecasts (i.e., $r_{t}=\bar{d}_{t}$ for all $t$ ) and that smaller values of $R E L$ indicate better reliability.

The third term on the RHS of (2) is the average squared difference between the subsample relative frequency $\left(\bar{d}_{t}\right)$ and the overall or sample relative frequency $(\bar{d})$. This term is a measure of the attributc resolution [see Murphy and Daan (1985)], and it will be denoted by RES. Note that $R E S=0$ for forecasts exhibiting no resolution (i.e., $\bar{d}_{t}=\bar{d}$ for all $t$ ) and that larger values of $R E S$ indicate better resolution. ${ }^{2}$

The skill of a sample of probability forecasts is generally defined as the average accuracy of the forecasts of interest relative to the average accuracy of forecasts produced by some 'standard of reference'. Since the sample relative frequency $(\bar{d})$ represents an estimate of the long-term base rate (or climatological probability), the former is a natural standard of reference in this context. Moreover, it provides the basis for the formulation of a sample skill score $S S$, where

$S S=\left(Q R^{*}-Q R\right) / Q R^{*}$,

or, from (2),

$S S=(R E S-R E L) / Q R^{*}$

[Murphy and Daan (1985)]. Note that $S S>(<) 0$ [i.e., skill is positive (negative)] when $R E S>(<)$ REL.

The measures of reliability, resolution, and skill described above relate to the entire sample of forecasts. Measures of these attributes can also be defined for each subsample, and these measures are expressed as follows:

$$
\begin{aligned}
& R E L_{t}=\left(r_{t}-\bar{d}_{t}\right)^{2}, \\
& R E S_{t}=\left(\bar{d}_{t}-\bar{d}\right)^{2}, \quad \text { and } \\
& S S_{t}=\left(R E S_{t}-R E L_{t}\right) / Q R^{*}
\end{aligned}
$$

Moreover, the accuracy of the forecasts in subsample t can be assessed using the quadratic score for these forecasts, $Q R_{t}$, where

$$
Q R_{t}=\left(r_{t}-\bar{d}_{t}\right)^{2}+\bar{d}_{t}\left(1-\bar{d}_{t}\right){ }^{3}
$$

2 Thus, resolution relates to the ability of forecasters to divide the sample into subsamples for which the respective relative frequencies differ from the overall (i.e., sample) relative frequency.

3 It might appear, by analogy with (2), that the expression for $Q R$, should contain a term relating to $R E S$. However, this term vanishes because the subsample and sample relative frequencies are both equal to $\bar{d}_{t}$ in this situation. 
Finally, note that

$$
\begin{aligned}
& R E L=(1 / K) \sum_{t=1}^{T} K_{t} R E L_{t}, \\
& R E S=(1 / K) \sum_{t=1}^{T} K_{t} R E S_{t}, \\
& S S=(1 / K) \sum_{t=1}^{T} K_{t} S S_{t}, \quad \text { and } \\
& Q R=(1 / K) \sum_{t=1}^{T} K_{t} Q R_{t} .
\end{aligned}
$$

The overall measures are the weighted averages of the subsample measures of the respective attributes. It should be noted that particular sets of values of $R E L_{t}, R E S_{t}, S S_{t}$, and $Q R$, are compatible with ranges of values of $R E L, R E S, S S$, and $Q R$, respectively, depending on the set of subsample frequencies $K_{t}(t=1, \ldots, T)$. Thus, the predictive distribution $K_{t} / K(t=1, \ldots, T)$ is an essential component of any method of evaluating probability forecasts.

\section{The attributes diagram}

\subsection{Basic framework}

The attributes diagram in two-event situations is a two-dimensional diagram with a cartesian coordinate system, in which the abscissa is the forecast probability $r_{t}$ and the ordinate is the relative frequency $\bar{d}_{t}$. In such a diagram, $\bar{d}_{t}$ plays the role of the dependent variable whose valucs are 'measured' for specific values of the independent variable $r_{t}$. The empirical 'curve' in this diagram consists of the $T-1$ line segments connecting the points $\left\{\left(r_{t}, \bar{d}_{t}\right) ; t=I, \ldots, T\right\}$ representing the $T$ subsamples of the sample of $K$ forecasts and observations. In addition to this curve, the predictive distribution $K_{t} / K(t=1, \ldots, T)$ is of considerable interest, and it is convenient to plot these relative frequencies (or simply the $K_{l}$ ) next to the respective points on the diagram.

The purpose of the attributes diagram is to provide a geometrical framework for comparing the empirical curve with various lines constituting sets of reference points with respect to specific attributes. For example, the locus of points $\bar{d}_{t}=r_{t}$ is the $45^{\circ}$ line in the diagram, which represents perfect reliability for each subsample of forecasts. The framework for the familiar reliability diagram consists solely of this line, together with the coordinate axes $r_{t}$ and $\bar{d}_{t}$ [e.g., see Murphy and Brown (1984), Murphy and Winkler (1984)].

Consideration of the other attributes leads to the identification of additional sets of reference points. For example, the locus of points $\bar{d}_{t}=\bar{d}$ is a horizontal line in the attributes diagram representing no resolution for each subsample of forecasts. Moreover, optimal (subsample) resolution is represented by the horizontal line segments $\bar{d}_{t}=0$ for $r_{t}<\bar{d}$ and $\bar{d}_{t}=1$ for $r_{t}>\bar{d} .{ }^{4}$

4 The phrase 'optimal resolution' is used here instead of 'perfect resolution' to emphasize the fact that these line segments represent the best values of resolution attainable given the existence of the respective subsamples (i.e., given the values of $r_{t}$ ). A similar comment applies to the phrases 'optimal skill' and 'optimal accuracy'. 


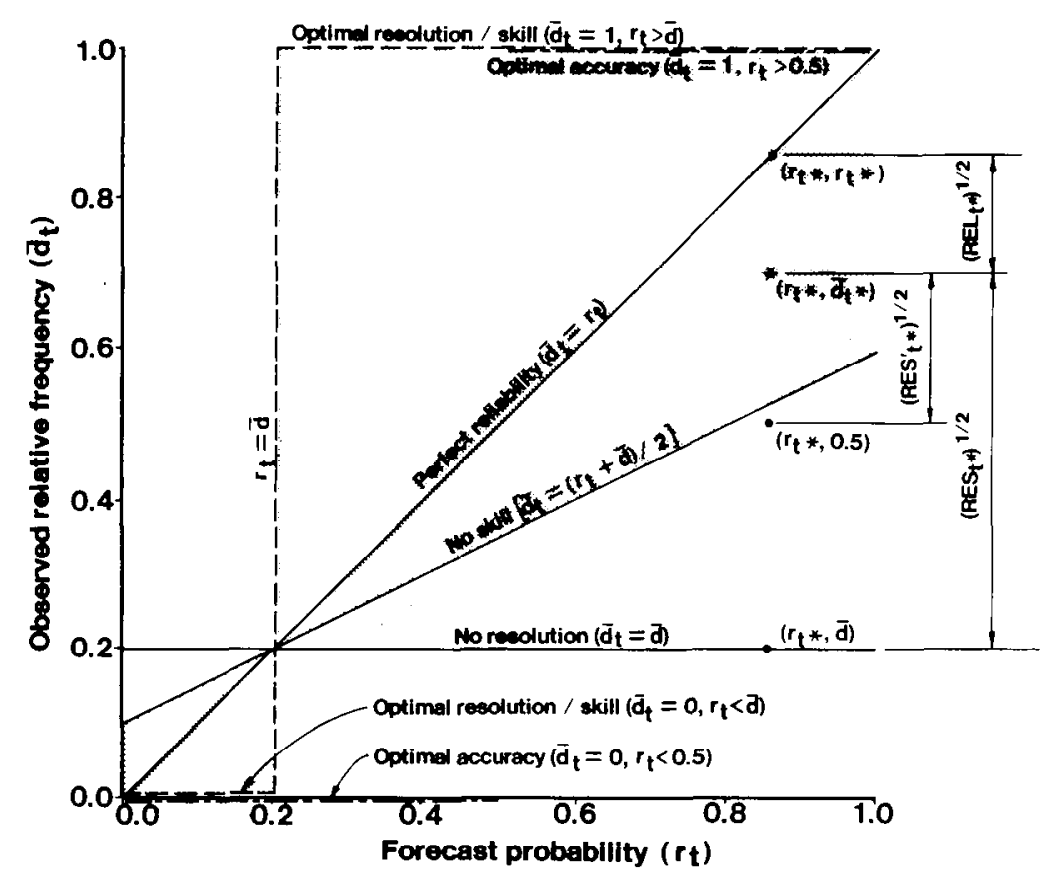

Fig. 1. The framework for the attributes diagram in two-event situations, with perfect reliability, no resolution, optimal resolution/skill, no skill, and optimal accuracy lines. An arbitrary point $\left(r_{t^{*}}, \bar{d}_{t^{*}}\right)$ on a hypothetical empirical curve is included to illustrate the measures of the respective attributes. The shaded region is the region of positive skill (see text for additional details).

Recall that $S S_{t}=0$ when $R E L_{t}=R E S_{t}$ [see eq. (7)]. Equating the expressions for $R E L_{t}$ and $R E S_{t}$ in (5) and (6), respectively, and solving for $\bar{d}_{t}$, we obtain the solution $\bar{d}_{t}=(1 / 2)\left(r_{t}+\bar{d}\right)$. This solution represents a no skill line equidistant between the $45^{\circ}$ line $\bar{d}_{t}=r_{t}$ and the horizontal line $\bar{d}_{t}=\bar{d}$. Moreover, $S S_{t}>(<) 0$ for $\bar{d}_{t}<(>)(1 / 2)\left(r_{t}+\bar{d}\right)$ when $r_{t}<\bar{d}$ and $S S_{t}>(<) 0$ for $\bar{d}_{t}>(<)$ $(1 / 2)\left(r_{t}+\bar{d}\right)$ when $r_{t}>\bar{d}$. It is quite easy to show that subsample skill increases linearly in the region of positive skill with decreasing (increasing) values of $\bar{d}_{t}$ when $r_{f}<(>) \bar{d} .{ }^{5}$ Thus, the horizontal line segments defining optimal resolution also represent lines of optimal (subsample) skill.

A schematic version of the attributes diagram is depicted in fig. 1 , in which the lines of perfect reliability, no resolution, optimal resolution, no skill, and optimal accuracy have been identified. In addition, the region of positive subsample skill scores is shaded (it has been assumed in constructing this diagram that $\bar{d}=0.2$ ). The diagram also contains an arbitrary point $\left(r_{t^{*}}, \bar{d}_{t^{*}}\right)$ (denoted by an asterisk) on the empirical curve for some hypothetical subsample of forecasts and observations. The distances between this point and the perfect reliability and no resolution lines are the square roots of the measures $R E L_{t}$ and $R E S_{t}$, respectively. Thus, the overall measures of these attributes, $R E L$ and $R E S$, are the weighted averages of these squared distances over all $T$ subsamples.

The interpretation of subsample accuracy $Q R_{t}$ in the attributes diagram is somewhat more complex. However, it should be noted that $Q R_{t}$ in (8) can be rewritten as follows:

$Q R_{t}=\left(r_{t}-\bar{d}_{t}\right)^{2}+(0.5)^{2}-\left(0.5-d_{t}\right)^{2}$.

5 Lines of equal skill in this diagram are hyperbolas with asymptotes $r_{t}=\bar{d}$ and $\bar{d}_{t}=(1 / 2)\left(r_{t}+\bar{d}\right)$ (we are indebted to Harald Daan for pointing out this fact). We have chosen not to show these curves in the attributes diagrams used here to avoid further complicating the geometrical displays. 


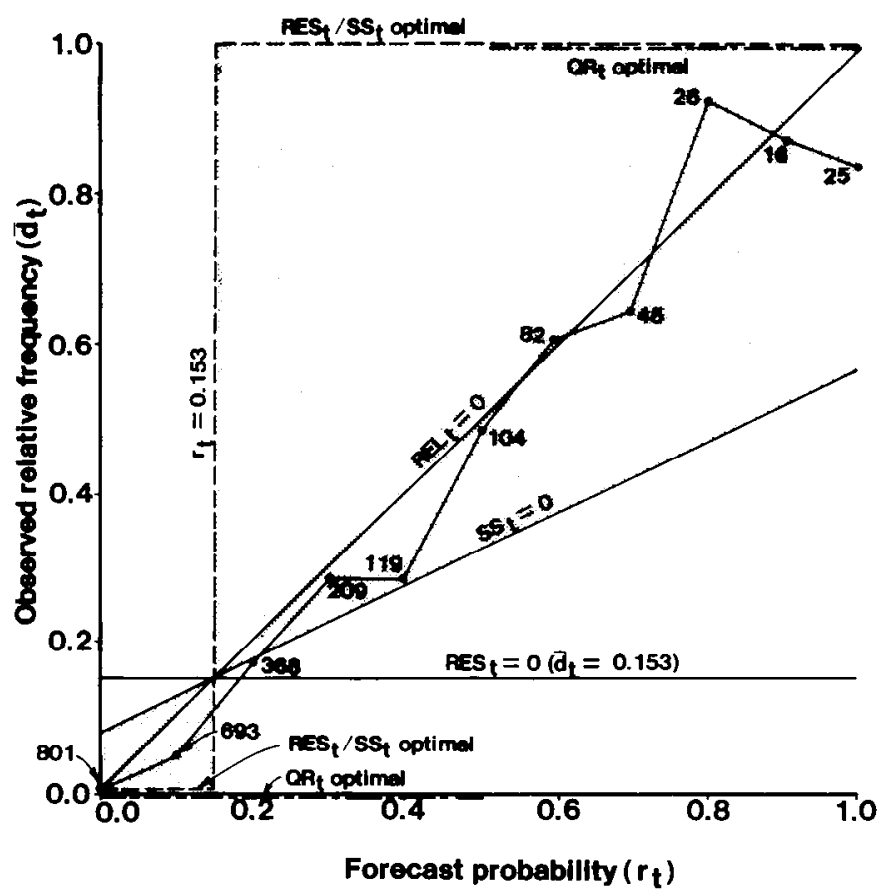

Fig. 2. An illustration of the attributes diagram, involving a sample of experimental precipitation probability forecasts with a threshold amount of 0.01 inches (see text for additional details).

Thus, subsample accuracy is the difference between two squared distances (aside from a constant): (a) the distance between the point $\left(r_{t^{*}}, \bar{d}_{t^{*}}\right)$ and the corresponding point $\left(r_{t^{*}}, r_{t^{*}}\right)$ on the perfect reliability line, and (b) the distance between the point $\left(r_{t^{*}}, \bar{d}_{t^{*}}\right)$ and the point $\left(r_{t^{*}}, 0.5\right)$ (we denote the square of this latter distance by $R E S_{t^{*}}^{\prime}$ ). Even the constant term in (13) can be viewed as a squared distance; namely, the distance between $\left(r_{t^{*}}, 0.5\right)$ and $\left(r_{t^{*}}, 0\right)$ [or $\left.\left(r_{t^{*}}, 1\right)\right]$. It can also be shown that subsample accuracy increases linearly with decreasing (increasing) values of $\bar{d}_{t}$ when $r_{t}<(>) 0.5$. Optimal subsample accuracy is represented by the lines $\bar{d}_{t}=0$ for $r_{t}<0.5$ and $\bar{d}_{t}=1$ for $r_{t}>0.5$. Finally, the overall measure of accuracy, $Q R$, is the weighted average of the 'sum' of squared distances in (13) over all $T$ subsamples [cf. (12)].

\subsection{Illustrations}

To illustrate the interpretation of the attributes diagram, we consider some samples of forecasts from a probabilistic quantitative precipitation forecasting experiment [Hsu (1982), Murphy et al. (1985)]. The empirical curve for a sample of forecasts - in which the threshold value defining the occurrence of precipitation is equal to 0.01 inches - is depicted in an attributes diagram in fig. 2. For these forecasts, $K=2488, T=11, r_{1}=0.0, \ldots, r_{11}=1.0$, and $\bar{d}=0.153$. Thus, the empirical curve consists of ten line segments joining the points $\left\{\left(r_{t}, \bar{d}_{t}\right) ; t=1, \ldots, 11\right\}$.

Comparison of the empirical curve with the perfect reliability line $\left(R E L_{t}=0: \bar{d}_{t}=r_{t}\right)$ indicates that the subsamples of forecasts generally are quite reliable, with only a slight tendency toward overforecasting [i.e., a tendency for forecast probabilities $\left(r_{t}\right)$ to exceed relative frequencies $\left(\bar{d}_{t}\right)$ ]. Moreover, comparison of this curve with the no resolution line $\left(R E S_{t}=0: \bar{d}_{t}=\bar{d}\right)$ reveals that most subsamples of forecasts possess considerable resolution. On the other hand, the distances between 


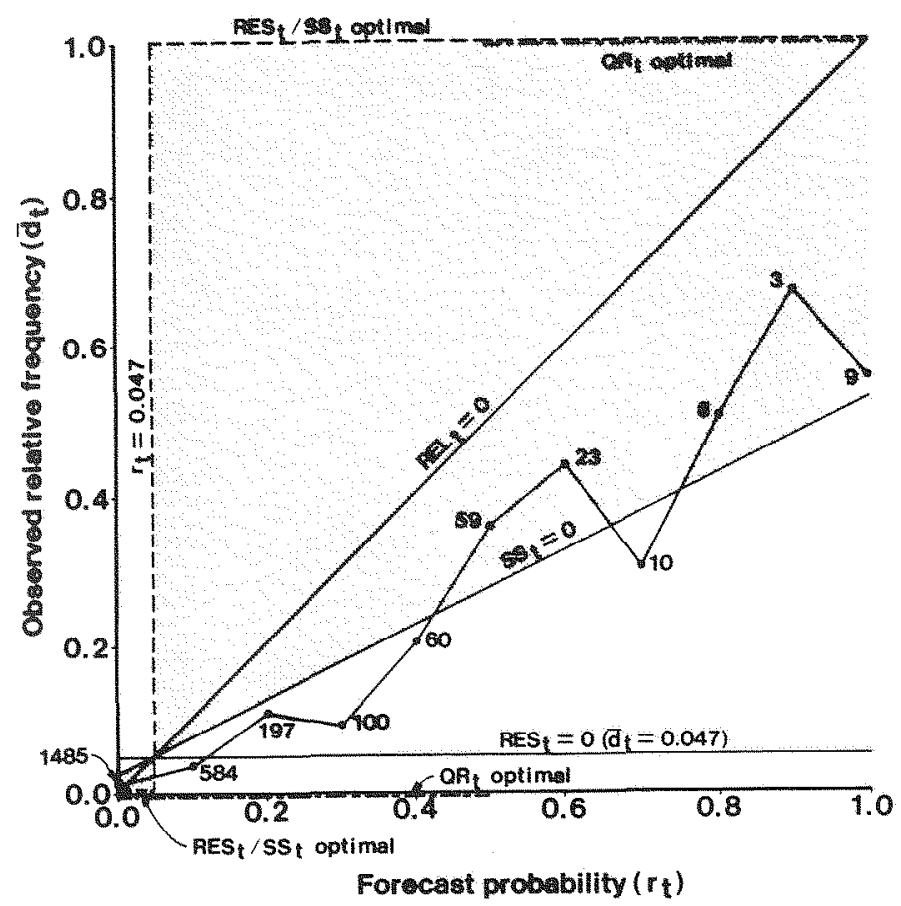

Fig. 3. An illustration of the attributes diagram, involving a sample of experimental precipitation probability forecasts with a threshold amount of 0.25 inches (see text for additional details).

the points defining the empirical curve and the optimal resolution/skill lines $\left(\bar{d}_{t}=0\right.$ for $r_{t}<0.153$ and $\bar{d}_{t}=1$ for $r_{t}>0.153$ ) are large, indicating that substantial improvements in resolution and skill are still possible. A similar comment could be made regarding the accuracy of the subsamples of forecasts. Nevertheless, this diagram also indicates that the skill of each subsample of forecasts is non-negative (i.e., $S S_{t} \geq 0$ for all $t$ ), thereby implying that the skill of the sample of forecasts as a whole is positive (in fact, $S S=0.32$ ). The subsample size $\left(K_{t}\right)$ appears next to each point on the empirical curve. These numbers reveal that small probability values are used much more frequently than large probability values. This predictive distribution is not surprising in view of the current state of the art of weather forecasting and the magnitude of the sample relative frequency of the event $(\bar{d}=0.153){ }^{6}$

Fig. 3 depicts the empirical curve for another sample of precipitation probability forecasts - in this case, the threshold value defining the occurrence of precipitation is equal to 0.25 inches and $\bar{d}=0.047$ [the values of $K, T$, and $r_{t}(t=1, \ldots, T)$ are the same as those for the previous sample]. Comparison of the curve with the perfect reliability line indicates that the subsamples of forecasts for this more extreme precipitation event exhibit considerable overforecasting, especially for large probability values. On the other hand, differences between points on the curve and the line $R E S_{t}=0$ reveal that the resolution of most subsamples of forecasts is quite substantial. The net effect is that the empirical curve tends to 'follow' the no-skill line, with approximately as many subsamples showing negative skill as showing positive skill. Overall, $S S=0.02$. Finally, the predictive distribution for these forecasts indicates that low probabilities are assigned to this event on a vast majority of

6 For more predictable events, the mode(s) of the predictive distribution would tend to migrate toward the extremes of the probability range. 
the 2488 forecasting occasions. Probabilities exceeding 0.5 are used on less than $2.2 \%$ of the occasions.

\section{Discussion and conclusion}

Attributes diagrams - and quantitative measures of the respective attributes such as the distance measures employed here - can be used to investigate the quality of samples of probability forecasts. These 'tools' provide a means of identifying the source(s) of any deficiencies in the forecasts - for example, a tendency toward overforecasting or underforecasting. ${ }^{7}$ Moreover, feedback concerning performance can be used to improve the quality of the forecasts. For example, in the first year of the Zierikzee experiment [Daan and Murphy (1982)], forecasters exhibited considerable overforecasting in their probability forecasts of wind speed, visibility, and precipitation events. At the end of this period, the forecasters were provided with extensive feedback concerning their performance, including individual and collective reliability diagrams, scores related to various attributes, etc. Subsequent evaluation of the results of this experiment for the second year [Murphy and Daan (1984)] revealed substantial improvements in the reliability and skill of the forecasts. As additional experience is acquired in the interpretation and use of attributes diagrams, they too may become quite useful in the diagnostic and feedback phases of probability forecasting.

The attributes diagram, as described in this paper, is applicable to two-event situations. However, many probability forecasting studies involve forecasts for multiple-event situations. We briefly describe two possible approaches to the problem of extending this geometrical framework to such situations. One approach - the so-called scalar approach - would consist of plotting the empirical curve for the relative frequencies and forecast probabilities associated with each event on a separate attributes diagram. As a result, $N$ diagrams would be produced in $N$-event situations. ${ }^{8}$ This approach should provide valuable insights into the extent to which forecast probabilities assigned to individual events possess certain desirable properties, but it ignores relationships among probabilities assigned to different events and therefore cannot be used to assess the overall performance of sets of probabilities.

Alternatively, a multi-dimensional attributes diagram could be employed to depict simultaneously the relative frequencies and forecast probabilities associated with the set of $N$ events. One such vector approach would make use of the fact that the sets of all possible relative frequencies and forecast probabilities in $N$-event situations constitute a $(N-1)$-dimensional simplex [e.g., see Murphy (1972b), Murphy and Daan (1985)]. This simplex is an equilateral triangle when $N=3$ and a regular tetrahedron when $N=4$, and it employs a barycentric coordinate system. The vectors representing the sets of relative frequencies and forecast probabilities are points in such a simplex, and the distances between these points are multi-dimensional analogues of the measure of reliability employed in this paper. ${ }^{9}$ It is also possible to identify hyperplanes in the simplex that represent standards of reference (e.g., a no-skill hyperplane). This approach has been investigated and applied in the $N=3$ situation by Hsu (1982). We believe that the vector approach generally is more

7 The sensitivity of decisions to 'errors' (e.g., miscalibration) in probability forecasts has been investigated recently by Henrion (1982). See also Fishburn et al. (1968).

8 Of course, since only $N-1$ relative frequencies and probabilities are independent, the empirical curves in the $N$ diagrams would be related. For example, application of the scalar approach in the two-event situation would yield two diagrams in which these curves would he mirror images of each other. This simple relationship between the curves would not hold in multiple-event situations.

9 In reality, the geometrical analogue of this particular vector approach in the two-event situation is the unit line segment an alternative framework to the two-dimensional attributes diagram cmployed in this paper. 
appropriate than the scalar approach, but it obviously is difficult to make full use of the former in situations in which the number of events exceeds three.

In this paper we have presented an attributes diagram that provides a geometrical framework for the description of the accuracy, reliability, resolution, and skill of probability forecasts in two-event situations. Moreover, these geometrical representations have been shown to be directly related to certain quantitative measures of the respective attributes. The interpretation of this framework has been illustrated by considering samples of forecasts from a probabilistic quantitative precipitation forecasting experiment. We believe that the attributes diagram will prove to be even more useful than the simple reliability diagram, and thus it should represent a valuable adjunct to quantitative assessments of the quality of probability forecasts based on scoring rules and other evaluation procedures.

\section{References}

Daan, Harald and Allan H. Murphy, 1982, Subjective probability forecasting in The Netherlands: Some operational and experimental results, Meteorologische Rundschau 35, 99-112.

Fischhoff, Baruch and Donald MacGregor, 1982, Subjective confidence in forecasts, Journal of Forecasting 1, $155-172$.

Fishburn, Peter C., Allan H. Murphy and Herbert H. Isaacs, 1968, Sensitivity of decisions to probability estimation errors: A re-examination, Operations Research 16, 254-267.

Henrion, Max, 1982, The value of knowing how little you know: The advantages of a probabilistic treatment of uncertainty in policy analysis, Ph.D. dissertation (Carnegie-Mellon University, Pittsburgh, PA).

Hsu, Wu-ron, 1982, Evaluation of a probabilistic quantitative precipitation forecasting experiment, M.S. thesis (Oregon State University, Corvallis, OR).

Lichtenstein, Sarah, Raruch Fischhoff and Lawrence D. Phillips, 1982, Calibration of probabilities: The state of the art to 1980, in: Daniel Kahneman, Paul Slovic and Amos Tversky, eds., Judgment under uncertainty: Heuristics and biases (Cambridge University Press, New York) 306-334.

Murphy, Allan H., 1972a, Scalar and vector partitions of the probability score: Part I, Two-state situation, Journal of $\Lambda$ pplied Meteorology 11, 273-282.

Murphy, Allan H., 1972b, Scalar and vector partitions of the probability score: Part II, N-state stituation, Journal of Applied Meterology 11, 1183-1192.

Murphy, Allan H., 1973, A new vector partition of the probability score, Journal of Applied Meteorology 12, 595-600.

Murphy, Allan H., 1985, Probabilistic weather forecasting, in: Allan H. Murphy and Richard W. Katz, eds., Probability, statistics, and decision making in the atmospheric sciences (Westview Press, Boulder, CO) 337-377.

Murphy, Allan H. and Barbara G. Brown, 1984, A comparative evaluation of objective and subjective weather forecasts in the United States, Journal of Forecasting 3, 369-393.

Murphy, Allan H. and Harald Daan, 1984, Impacts of feedback and experience on the quality of subjective probability forecasts: Comparison of results from the first and second years of the Zierikzee experiment, Monthly Weather Review $112,413423$.

Murphy, Allan H. and Harald Daan, 1985, Forecast evaluation, in: Allan H. Murphy and Richard W. Katz, eds., Probability, statistics, and decision making in the atmospheric sciences (Westview Press, Boulder, CO) 379-437.

Murphy, Allan H. and Robert L. Winkler, 1977, Reliability of subjective probability forecasts of precipitation and temperature, Applied Statistics 26, 41-47.

Murphy, Allan H. and Robert L. Winkler, 1984, Probability forecasting in meteorology, Journal of the American Statistical Association 79, 489-500.

Murphy, Allan H., Wu-ron Hsu, Robert L. Winkler and Daniel S. Wilks, 1985, The use of probabilities in subjective quantitative precipitation forecasts: Some experimental results, Monthly Weather Review 113, 2075-2089.

Sanders, Frederick, 1963, On subjective probability forecasting, Journal of Applied Meteorology 2, $191-201$.

Wallsten, Thomas S. and David V. Budescu, 1983, Encoding subjective probabilities: A psychological and psychometric review, Management Science 29, 151-173

Winkler, Robert L. and Allan H. Murphy, 1968, 'Good’ probability assessors, Journal of Applied Meteorology 7, 751-758.

Yates, J. Frank, 1982, External correspondence: Decompositions of the mean probability score, Organizational Behavior and Human Pcrformance 30, 132-156. 